\title{
VIVRE À LA MERCI
}

Le care et les trois figures de la vulnérabilité dans les théories politiques contemporaines

Estelle Ferrarese

Association Multitudes | « Multitudes »

2009/2 n 37-38 | pages 132 à 141

ISSN 0292-0107

ISBN 9782354800543

Article disponible en ligne à l'adresse :

http://www.cairn.info/revue-multitudes-2009-2-page-132.htm

\section{Pour citer cet article :}

Estelle Ferrarese, « Vivre à la merci. Le care et les trois figures de la vulnérabilité dans les théories politiques contemporaines », Multitudes 2009/2 ( $n^{\circ} 37-38$ ), p. 132-141.

DOI $10.3917 /$ mult.037.0132

Distribution électronique Cairn.info pour Association Multitudes.

(C) Association Multitudes. Tous droits réservés pour tous pays.

La reproduction ou représentation de cet article, notamment par photocopie, n'est autorisée que dans les limites des conditions générales d'utilisation du site ou, le cas échéant, des conditions générales de la licence souscrite par votre établissement. Toute autre reproduction ou représentation, en tout ou partie, sous quelque forme et de quelque manière que ce soit, est interdite sauf accord préalable et écrit de l'éditeur, en dehors des cas prévus par la législation en vigueur en France. Il est précisé que son stockage dans une base de données est également interdit. 


\section{Vivre à la merci \\ Le care et les trois figures de la vulnérabilité dans les théories politiques contemporaines \\ Estelle Ferrarese}

Tout comme les théories du care, celles de la reconnaissance, certaines théories néorépublicaines rompant avec leur tradition, Judith Butler raisonnant sur l'idée de vies précaires, Giorgio Agamben ramenant la biopolitique foucaldienne à l'idée du gouvernement de corps tuables, Martha Nussbaum pensant la fragilité de la vie bonne, (la liste n'étant pas close), témoignent d'une résurgence du thème de la vulnérabilité, corporelle et morale, comme problème politique et moral en soi. Tous évoquent la vulnérabilité non pas pour ce qu'elle prévient, empêche, ou gêne d'accès à d'autres biens ou fins, mais en tant qu'elle est constitutive.

En ce sens, elles répondent à l'urgence politique de contrer la figure du sujet autoengendré et autosuffisant, jusque dans ses nouvelles incarnations. Idée protéiforme d'empowerment, théories de l'identité pariant sur une fière assertion du sujet, paradigme, désormais prégnant dans les sciences sociales, de la négociation des parcours de vie et des préférences: la décomposition de la subjectivité politique marxiste et les paradoxes des structures postmodernes ont laissé le champ libre à l'évidence d'un sujet qui ne peut certes se choisir librement, mais qui est capable de se constituer en manager de luimême, assurant sinon rationnellement, du moins habilement, la somme arithmétique de ses possibilités et de ses facettes identitaires. C'est une anthropologie radicalement antithétique qui se dessine avec les réflexions contemporaines sur la vulnérabilité.

Cette anthropologie doit être la plus fine possible. Ainsi Axel Honneth en appelle à une «anthropologie atténuée et formelle ${ }^{1}$. En outre, le fait que cette vulnérabilité soit constitutive est interprété comme un argument illégitime pour la placer hors champ politique. Tout d'abord, il est évident que la vulnérabilité n'existe qu'en situation, fût-elle celle de l'absence d'organisation que serait l'état de nature. En même temps, si soutenir cela ne pose pas de gros problèmes conceptuels, la naturalisation de

1 Axel Honneth, La société du mépris, Paris, La Découverte, 2006, p. 99. 


\section{Majeure Politiques du care}

la vulnérabilité par la référence à une fragilité ontologique est particulièrement meurtrière d'un point de vue politique, frappant du sceau de la naïveté ou de la confusion épistémologique tout énoncé visant à la parer. Elle concourt en outre à légitimer l'organisation existante de cette vulnérabilité. En effet, les institutions protègent toujours, dans leur agencement même, certains et en exposent d'autres à différentes formes de torts. De ce point de vue, penser une vulnérabilité constitutive implique aussi d'inverser la relation traditionnelle entre le problème politique et le problème anthropologique; si l'institution humaine se donne pour fondement et justification une nature humaine, elle concourt à la produire. Comme le relève Guillaume Le Blanc que l'anthropologie de la vulnérabilité ait succédé à celle de la mortalité signifie aussi et surtout que se met en place un nouvel art de gouverner les vivants ${ }^{2}$.

Parler d'anthropologie de la vulnérabilité n'empêche alors pas de penser des vulnérabilités construites en abyme, c'est-à-dire qu'une vulnérabilité universellement partagée se trouve doublée pour certains d'une susceptibilité forte du tort.

On peut même soutenir, comme le fait Philip Pettit, l'idée que l'écrasement d'une vulnérabilité sur l'autre, c'est-à-dire l'émergence d'une classe unique de vulnérabilité, loin d'être première, constitue un horizon politique en soi, au sens où il s'agirait de penser une société où l'appartenance à une caste, à une classe, à un genre, perde sa fonction d'indicateur d'une vulnérabilité particulière ${ }^{3}$.

Relevons, pour finir, que la vulnérabilité telle que je l'envisage dans cet article n'est pas synonyme "d'une vie jamais assurée de son maintien, s'essayant sans assurance à ajourner le négatif [...] de tout un spectre de terreurs et d'ombres qui peuvent emporter la vie et la faire disparaître» ${ }^{4}$. Ici, la menace n'est jamais endogène, elle provient nécessairement de l'autre (compris comme singulier, collectif, ou comme structure généralisée). S'il existe une vulnérabilité à la maladie, à l'accident, inhérente à la fragilité des structures organiques et/ou à leur maturation ou leur dégénérescence, je la nomme précarité, afin de me concentrer sur la vulnérabilité comme possibilité de l'infliction d'un tort ou de l'absence de soin (lequel peut être rendu nécessaire par l'expérience de la précarité), bref la vulnérabilité de l'homme relationnel, la vulnérabilité à autrui. Je tenterai ici de mettre en évidence trois pôles thématiques qui structurent cette résurgence de l'idée de vulnérabilité: le modèle d'une disponibilité à la blessure physique et morale, celui d'une association stricte de l'idée de vulnérabilité au concept de dépendance (qu'illustrent tout particulièrement les théories du care), et enfin la vulnérabilité comme impropriété de soi.

\section{La disponibilité à la blessure}

Les théories hégéliennes contemporaines de la reconnaissance révèlent sans ambiguïté une idée de vulnérabilité morale pensée par homologie avec la vulnérabilité corporelle; de même que le corps humain est à chaque instant disponible à la blessure physique, la psyché est constamment ouverte au tort et à la blessure morale. Elles invitent à conce-

2 Guillaume Le Blanc, Vies ordinaires, vies précaires, Paris, Seuil, 2007, p. 246.

3 Philip Pettit, Républicanisme. Une théorie de la liberté et du gouvernement, Paris, Gallimard, 2004, p. 165.

4 Guillaume Le Blanc, op. cit., p. 168. 


\section{Multitudes37}

voir une exigence de préservation de l'intégrité psychique de manière analogue à la préservation de l'intégrité physique. Parce qu'il détruit une présupposition essentielle de la capacité de l'individu à agir, le déni de reconnaissance est qualifié par Axel Honneth de blessure morale; il est une atteinte portée à une entité qui s'en trouve altérée, mutilée. Quant à la psyché et à l'autonomie, elles se trouvent donc livrées sans contrôle à la volonté d'autrui.

Jürgen Habermas parle ainsi de manière répétée de «l'extrême vulnérabilité» de la personne, qui provient de ceci qu'elle «ne forme un centre intérieur que dans la mesure où elle s'aliène en même temps à des relations interpersonnelles produites intersubjectivement. Ainsi s'explique une mise en péril presque constitutionnelle», une faiblesse chronique de l'identité "qui pré-existe même à la vulnérabilité manifeste de l'intégrité du corps et de la vie» ${ }^{5}$. Il en résulte une idée de vulnérabilité relativement étroite; d'une part, elle est ramassée autour de la possibilité d'une atteinte. D'autre part, la vulnérabilité ne constitue pas un bien, comme c'est le cas dans la tragédie grecque lue par Martha Nussbaum dans The Fragility of Goodness', c'est un fait.

L'on retrouve une approche similaire dans les nombreuses occurrences de l'idée de vulnérabilité repérables dans les écrits récents de Judith Butler. Usant précédemment du terme, notamment dans Excitable Speech, pour rendre compte du fait que l'adresse de l'autre est ce qui inaugure la possibilité d'une puissance d'agir, elle l'a récemment rendu synonyme de destructibilité; ce qu'elle nomme indistinctement vulnérabilité et précarité est disponibilité à la blessure et à l'annihilation, physique et psychique: «Perte et vulnérabilité viennent de ce que nous sommes des corps socialement constitués, attachés aux autres, menacés de perdre ces attachements, exposés aux autres, menacés de violence du fait de cette exposition ${ }^{7}$.

Butler cherche néanmoins à échapper au spectre d'une anthropologie sous-jacente à son travail, par trop en contradiction avec les prémisses de sa théorie de la performativité, qui postulait une capacité à agir qui émerge, sans que rien ne la précède. Pour ce faire, elle use d'une sorte de boucle théorique: «La vulnérabilité dépend fondamentalement des normes existantes de reconnaissance si elle doit être attribuée à un sujet humain ${ }^{8}$. En réalité, en pointant des dimensions irréductibles de la socialité humaine, la violabilité et l'affectabilité, elle ne peut éviter de supposer une vie corporelle préalable à son émergence dans le langage: «Qui dit corps dit mortalité, vulnérabilité, et puissance d'agir: la peau et la chair nous exposent au regard et au contact des autres comme à leur violence ". Tout en soulevant la question de ce qui est défini comme vie, et en soulignant qu'une rencontre d'une vie dans sa précarité n'est pas quelque chose qui advient, mais qui est socialement construit, Butler ne peut échapper ni au vocabulaire, ni à la perspective d'un corps précédant et presque résistant, dans son affectabilité, à sa reconnaissance et à sa constitution en corps humain digne ou non d'être aimé et pleuré.

5 Jürgen Habermas, De l'éthique de la discussion, Paris, Cerf, 1992, p. 67.

6 Martha Nussbaum, The Fragility of Goodness, Cambridge, Cambridge University Press, 1986, p. 2.

7 Judith Butler, Vie Précaire, Paris, éditions Amsterdam, 2005, p. 46.

8 Judith Butler, Giving an Account of Oneself, New York, Fordham University Press, 2005, p. 43.

9 Judith Butler, Vie Précaire, op. cit., p. 52. 


\section{Majeure Politiques du care}

Les obligations morales qui découlent de cette approche sont posées par les différents auteurs, par-delà les différences d'univers philosophiques, dans le vocabulaire de la protection. Ainsi Habermas écrit-il: «J'aimerais nommer 'morales' toutes celles des intuitions qui nous informent sur la question de savoir comment nous devons nous comporter au mieux afin de contrecarrer l'extrême vulnérabilité des personnes, en la protégeant et en l'épargnant $\rangle^{10}$. Chez Honneth, aux attentes fondamentales de reconnaissance du sujet correspondent autant de devoirs de reconnaissance pesant sur les personnes susceptibles d'y répondre, sous la forme d'attitudes qu'elles sont mutuellement obligées d'adopter pour garantir ensemble les conditions de leur intégrité psychique. La morale est ainsi définie comme « une institution collective pour garantir notre intégrité personnelle $»^{11}$.

Le thème de la vulnérabilité porte avec lui chez Judith Butler aussi la nécessité de réagir aux différentes formes d'atteintes violentes à l'intégrité corporelle et la sorte d'autonomie qu'elle rend possible, et donc de devenir l'objet d'une politique et d'une éthique. Cette vulnérabilité partagée se retrouve même instituée en socle qui tient, ou devrait tenir, les hommes ensembles, fondant un impératif minimal de solidarité. Tout comme chez Levinas, le fait que l'autre, de par sa vulnérabilité, se trouve dans un état de nudité totale, d'absence absolue de défense, vis-à-vis de ma destructivité, fonde des exigences éthiques de ne pas porter atteinte à l'intégrité dont la persistance est placée entre mes mains. En d'autres termes, l'anthropologie de la vulnérabilité présente dans l'œuvre de Butler fonde une éthique de la non-violence.

Les institutions rendues nécessaires et légitimes par cette assomption d'une disponibilité à la blessure doivent quant à elles parer à l'absence de protection de cet être humain toujours objet possible d'effraction.

Si ce type de raisonnement a pu produire une tradition réactionnaire, incarnée par exemple par Arnold Gehlen, il amène plutôt les théories qui nous préoccupent à appréhender le droit dans sa fonction protectrice. C'est bien sûr là une conception qui s'inscrit sans ambiguïtés dans la tradition libérale. L'obligation négative de ne pas infliger un tort y est bien installée dans le droit (tandis qu'un devoir positif de venir en aide aux autres est rarement enjoint).

Ainsi Honneth conçoit la solidification juridique du contenu normatif de la reconnaissance à une période donnée comme une extension des droits subjectifs négatifs. L'idée d'un bouclier de droits est d'ailleurs déclinée sous de multiples formes au sein des théories de la reconnaissance, de celle d'un bouclier de droits collectifs chez Charles Taylor, qui invoque des protections étatiques et différenciées pour les «sociétés distinctes", à celle d'un bouclier de droits limité à des cas où la faiblesse est exposée à une force excessive, dans le cas de Nancy Fraser.

Cette conception défensive du droit permet de poser la question de la prévention et de la sanction du tort, sans céder à l'idée d'un droit à la reconnaissance, sous l'espèce d'un droit fondamental entendu comme possibilité d'élever des revendications dont la satisfaction sociale est considérée comme justifiée (bien que ces théories, et

10 Jürgen Habermas, De l'éthique de la discussion, op. cit., p. 19. C'est moi qui souligne.

11 Axel Honneth, "Between Aristotle and Kant - Sketch of a Morality of Recognition ", in Advances in Psychology, 137, 2005: «Morality in Context», p. 51. 


\section{Multitudes37}

celle de Honneth en particulier, ne soient pas dénuées d'ambiguïtés sur ce point). Chez Butler, le langage des droits n'est désormais plus exclu (elle parle par exemple de droits garantissant l'intégrité corporelle et la libre disposition de soi). Néanmoins le ressort politique de son propos sur la vulnérabilité est plutôt à chercher dans la volonté qu'elle manifeste d'étendre le spectre des vies qui sont considérées comme des vies. Son propos selon lequel une vie n'est une vie que pour autant qu'elle a été reconnue telle implique que l'attitude éthique ne surgit pas hors des cadres d'interprétation quotidiens, et qu'il entre dans la visée politique de sa propre théorie de combattre les conditions qui font que certaines vies sont plus exposées que d'autres, rejoignant ainsi l'horizon politique d'une seule classe de vulnérabilité comme précédemment avec Philip Pettit.

Je souhaiterais à présent me pencher sur la théorie de Giorgio Agamben, qui est à l'inverse dénuée de visée éthique, mais définit l'essence de la politique comme étant de répondre à la vulnérabilité corporelle des êtres humains. Elle part d'une idée du corps humain appréhendé d'abord dans sa destructibilité, ce qui suppose pour Agamben d'abandonner de l'idée foucaldienne de biopouvoir le gouvernement de la population par la naissance ou encore le dressage des corps.

Au cœur de l'agencement théorique d'Agamben se trouve la notion de «vie nue», définie par opposition à ce qu'il appelle la «forme-de-vie», une vie qui ne peut jamais être séparée de sa forme. $\mathrm{La}$ « vie nue» est ce sur quoi naît et s'acharne le pouvoir, à l'exemple paroxystique du camp, dont les habitants ont été dépouillés de tout statut politique et réduits intégralement à la vie nue, et qui est l'espace "où le pouvoir n'a en face de lui que la pure vie biologique sans aucune médiation ${ }^{12}$, des «corps besogneux et exclus $»^{13}$, tuables. D'une part, l'État-nation fait donc de la pure vie humaine le fondement de sa souveraineté. D'autre part, la vie nue est conservée et protégée seulement dans la mesure où elle se soumet au droit de vie et de mort du souverain ou de la loi.

Mais il ne s'agit pas simplement d'une variante du raisonnement hobbesien. Non seulement, avec le souverain vient autant la sécurité (ou en tout cas la régulation de la vulnérabilité) que le statut politique, mais Agamben pose de surcroît: «L'exposition est le lieu de la politique. S'il n'existe pas, peut-être, de politique animale, c'est uniquement parce que les animaux, qui vivent sans cesse dans l'ouvert, ne cherchent pas à s'approprier leur exposition et restent simplement en elle sans s'en soucier [...] Ainsi, il transforme l'ouvert en un monde, dans le champ d'une lutte politique sans quartiers» ${ }^{14}$. Ce n'est donc pas seulement le concept de gouvernement ni celui d'État, mais celui de politique qu'Agamben déduit de l'idée de vulnérabilité, proposant par là l’un des énoncés les plus forts sur le sens de l'organisation collective parmi les théories contemporaines.

\section{Vulnérabilité et dépendance}

L'idée de disponibilité à la blessure permet une distinction forte entre vulnérabilité et dépendance, la première renvoyant plutôt à l'idée d'indétermination, d'ouverture au monde, tandis que la dépendance se trouve toujours au seuil de la subordination, donc

12 Giorgio Agamben, Moyens sans fins, Notes sur la politique, Paris, Rivages Poche, 2002, p. 51.

13 Ibid., p. 41.

14 Ibid. p. 105. C'est moi qui souligne. 


\section{Majeure Politiques du care}

de la détermination. Dans cette perspective, il peut exister des phénomènes de passage à la dépendance permettant d'échapper à la vulnérabilité. C'est notamment ce qui fonde le pouvoir politique chez Agamben, en ce sens que l'apparition du souverain, dont l'intégrité physique des sujets dépend, met fin à une fragilité à la merci de tous les possibles.

En revanche, les deux concepts de vulnérabilité et de dépendance tendent à être confondus dans les théories du care; ainsi de ce passage de Moral Boundaries, de Joan Tronto, où l'on glisse de l'un à l'autre sans médiation: «La vulnérabilité a des conséquences morales sérieuses. [...] Tout au long de notre vie, nous passons tous par des degrés variés de dépendance et d'indépendance, d'autonomie et de vulnérabilité ${ }^{15}$. Ici, la vie est suspendue non pas à une abstention, mais à un geste que celui qui est menacé ne peut accomplir lui-même. La survie, ou le maintien d'une relation positive à soi, dépend de la réalisation d'une obligation positive, de la réalisation d'un soin, d'un acte. D'où ce lien à la dépendance, explicité par Patricia Paperman comme «dépendance à l'égard des fournisseurs de soin $»^{16}$.

Cette formulation permet de renvoyer à des torts infligés par l'inaction des autres plutôt que par leur action, ce que proscrit la métaphore de la blessure.

La vulnérabilité entendue dans ce sens implique alors de rendre moralement équivalents les actes et les omissions, les obligations négatives et positives, pour reprendre les termes du programme que se donne Robert Goodin dans Protecting the $V_{u l n e r a b l e}{ }^{17}$. L'on n'est plus simplement vulnérable aux actions d'un autre violent, ou humiliant, mais à tous ces autres qui ne nous viennent pas en aide; il est ainsi possible de conceptualiser une vulnérabilité à des «amis riches » ${ }^{18}$, au sens où l'on est vulnérable à la possibilité qu'ils ne nous prêtent pas secours alors que nous sommes dans le besoin. Le tort à la susceptibilité duquel renvoie le terme de vulnérabilité se ramène alors moins à la métaphore de la blessure qu'à celle de l'abandon. Carol Gilligan pose ainsi, à côté des injonctions morales qui sont celles des théories traditionnelles de la justice, «l'injonction morale, de ne pas abandonner, de ne pas agir de façon inconsidérée et négligente, ne pas trahir, y compris vous-même ${ }^{19}$.

On ne trouve pas ici, ou pas de manière prégnante, de vocabulaire de la protection. Le problème moral et politique principal n'est pas posé par celui qui s'apprête à infliger un tort, une blessure, mais par celui qui refuse de devenir caregiver $^{20}$, qui s'exempte de toute responsabilité, qui s'exclue du groupe des personnes entre lesquelles sont distribuées les responsabilités, ce que Joan Tronto appelle l'irresponsabilité du privilégié. Il existe en effet une différence fondamentale, par rapport au précédent modèle, dans l'allocation de la responsabilité: dans le cas de la vulnérabilité comme disponibilité à la blessure, la responsabilité causale coïncide avec la responsabilité d'action, au

15 Joan Tronto, Moral Boundaries. A Political Argument for an Ethic of Care, NY, Routledge, 1993, p. 135.

16 Patricia Paperman, «Les gens vulnérables n'ont rien d'exceptionnel», in Patricia Paperman et Sandra Laugier ed. Le souci des autres, Paris, Éditions de l'EHESS, 2005, p. 289.

17 Robert E. Goodin, Protecting the Vulnerable. A Reanalysis of Our Social Responsabilities, University of Chicago Press, 1985, p. 25 sq., éd. franç. Un monde vulnérable, La découverte, 2009.

18 Ibid., p. 88.

19 Carol Gilligan, «Une voix différente. Un regard prospectif à partir du passé», conférence, EHESS,13 mai 2009.

20 Joan Tronto, «Global Justice for Global Care Workers: Some Limits to Recognition and Redistribution ", Paper for APSA Meeting, 2008. 


\section{Multitudes37}

sens où il appartient d'abord à celui qui est en mesure de porter une atteinte fatale de suspendre son geste.

Ici, la présence massive du mot besoin modifie la constellation. En effet, que $\mathrm{A}$ ait besoin de $\mathrm{X}$ signifie simplement que $\mathrm{A}$ devrait obtenir $\mathrm{X}$, sans que l'on précise de qui, et donc qui est responsable. L'on trouve de fait, dans les théories qui posent la vulnérabilité comme dépendance, l'idée que le niveau de responsabilité augmente avec la capacité à affecter (Goodin), ou encore des raisonnements multiples autour de l'idée de responsabilités spéciales, liées à l'existence de relations personnelles spécifiques, à notre condition «d'être attaché» (Feder Kittay).

Néanmoins, ce que nous apprennent les théories du care, c'est que la volatilité de l'allocation de la responsabilité impliquée par cette forme de vulnérabilité est à l'origine d'une série d'arrangements historiques qui laissent place à la domination, qu'ils prennent la forme de sédimentation de rôles de genre (avec l'assignation traditionnelle du travail de soin et de l'affect de la sollicitude aux femmes) ou de structures économiques supposant des filières de migration, qui assurent la réalisation du travail de soin par une main d'oeuvre mal rémunérée et docile. Elles démasquent le dispositif suivant: nos sociétés partagent l'idée d'un droit, à ce que des besoins fondamentaux soient comblés, sans que l'argumentation ou la certitude qui fonde ce droit ne résolve - ne puisse résoudre - la question de son pourvoyeur.

La responsabilité échoue finalement à celui qui perçoit le besoin. Dès lors, il n'y a qu'à ne pas percevoir, pour ne pas être enjoint. La catégorie d'attentiveness, centrale aux théories du care, qui renvoie à une capacité à percevoir et à répondre à un besoin ou à une souffrance, et dont Tronto dit que l'éthique du care traite l'absence comme une forme de «mal moral $»^{21}$ est à comprendre comme dotée d'une portée critique. Il suffit en effet de couler les schémas de perception qui rendent visible le besoin dans les attributs du genre féminin pour entrelacer acquittement d'une obligation morale et subordination politique. Desserrer cette étreinte, sans en créer de nouvelles, tel est le cœur de la réflexion des théories de la vulnérabilité comme dépendance.

\section{L'impropriété de soi}

Les théories d'une vulnérabilité constitutive postulent l'impossibilité de l'auto-suffisance, ou une forme d'impropriété de soi. Dans le cas des théories de la reconnaissance, le maintien de soi dépend de fait de l'abstention de certains gestes; ou pour le dire autrement, l'autre est ma condition de possibilité, ce qui est également l'assomption fondatrice de l'éthique du care: «dans la perspective du care, le soi et les autres ne sont pas représentés comme des entités séparées: la relation constitue la figure centrale à partir de laquelle le sujet/agent moral perçoit le besoin et répond à cette perception. $»^{22}$ Plus généralement, la vulnérabilité constitue la limite constitutive de mes pouvoirs, jusqu'à rendre compte du fait que la vie s'échappe à elle-même en permanence, qu'elle n'est jamais retenue dans les contours d'un «je» qui la maîtrise.

21 Joan Tronto, Moral Boundaries, op. cit., p. 127.

22 Sandra Laugier, Patricia Paperman, «Présentation», in Carol Gilligan, Une voix différente, Paris, Flammarion Champs, 2008, p. XXIII. 


\section{Majeure Politiques du care}

Cette impropriété de soi est parfois simplement célébrée; c'est la perspective adoptée par Butler à l'époque d'Excitable Speech, où la puissance d'agir émerge de la scène constituée par une "vulnérabilité habilitante», l'adresse de l'autre étant ce qui inaugure la possibilité d'une puissance d'agir.

La fragilité qui afflige la capacité humaine d'agir est perçue comme problématique, lorsqu'elle l'est, sous deux aspects différents: soit la vulnérabilité devient quasiment synonyme de contingence, soit elle signifie susceptibilité de la subordination et de la domination.

La première forme est celle dont se préoccupe Martha Nussbaum, dans son idée d'une exposition au sort, aux "circonstances", qui brosse la perspective d'une dépendance aveugle et douloureuse à ce qui peut survenir. La seule maîtrise qu'il reste à l'être humain réside dans la possibilité de redoubler sa propre vulnérabilité: «En assignant une valeur à l'amitié dans notre conception de la vie bonne, nous nous rendons plus vulnérables à la perte. Nous nous rendons également sensibles à des pertes qui, à proprement parler, ne sont pas les nôtres. Une personne dépourvue d'attachements forts n'a qu'à se soucier de sa propre santé, de sa propre vertu, de sa propre réussite $»^{23}$. Philip Pettit produit pour sa part une réflexion sur la seconde forme, la concentrant sur le fait d'avoir à vivre d'une façon qui nous expose à des maux que cet autre est en position de nous infliger arbitrairement. Il définit le républicanisme comme caractérisé par la conviction qu'une personne est privée de liberté dès lors que d'autres ont sur elle un pouvoir arbitraire d'interférence, qu'ils en fassent usage ou non.

Dans le cas de la disponibilité à la blessure, vulnérabilité corporelle et vulnérabilité morale s'articulent par homologie. Elles relèvent plutôt d'un continuum dans les théories de la dépendance; Tronto écrit ainsi: «le care est une activité caractéristique de l'espèce humaine qui inclut tout ce que nous faisons en vue de maintenir, de continuer ou de réparer notre «monde» de telle sorte que nous puissions y vivre aussi bien que possible. Ce monde inclut nos corps, nos individualités (selves), et notre environnement, que nous cherchons à tricoter ensemble dans un maillage complexe qui soutient la vie $»^{24}$. Dans le troisième modèle, le corps possède une moindre force. La vulnérabilité ainsi entendue implique qu'un tort menace le bien ou les «intérêts» de la personne, l'intégrité du corps figurant parmi ces «intérêts» sans se voir attribuer un statut particulier. Ainsi Nussbaum perçoit chez Euripide la conscience d'une vulnérabilité exacerbée des femmes au sort. Cette vulnérabilité au sort s'exprime dans une exposition toute particulière aux atteintes corporelles: «Ce sont les femmes qui sont violées et capturées pour être transformées en esclave en temps de guerre, alors que les hommes ont une chance de mourir bravement. $»^{25}$ La vulnérabilité physique est ainsi interprétée dans les termes d'une impuissance devant les affronts de la guerre, de la mort, de la trahison.

Dans la mesure où l'invulnérabilité n'est jamais présentée comme une alternative (précisément puisque que tous ces penseurs partent d'une anthropologie de la vulnérabilité), la réflexion sur l'organisation collective a recours au vocabulaire de la limite, ou du seuil; il s'agit de définir le point à partir duquel la vulnérabilité devient

23 Martha Nussbaum, The Fragility of Goodness, op. cit., p. 361.

24 Joan Tronto, Moral Boundaries, op. cit., p. 103.

25 Martha Nussbaum, The Fragility of Goodness, op. cit., p. 413. 


\section{Multitudes37}

moralement problématique et objet légitime d'intervention politique.

C'est le sens que l'on peut conférer à la mise en place de sa théorie des capabilités par Nussbaum, qui établit une liste des capabilités centrales permettant de distinguer entre les "circonstances» auxquelles sont confrontés les êtres humains, en fonction de ce qui produit cette vulnérabilité et de ce qui est affecté. Les capabilités, explicitement associées à l'idée de «niveau seuil», constituent à leur tour « une base pour les principes constitutionnels fondamentaux que les citoyens sont en droit d'exiger de leur gouvernement $»^{26}$.

L'on pourrait encore une fois citer Goodin et sa conviction qu'une relation de dépendance devient moralement problématique dès lors que la capacité de retrait de la relation, pour la partie subordonnée, est inexistante ou menacée ${ }^{27}$.

Enfin, sur le plan politique, l'on peut identifier deux grandes possibilités face à ce type de vulnérabilité: garantir à chaque partie la possibilité de se défendre contre l'autre, ou bien prévenir la menace d'exploitation, en bridant celui qui est en position de tirer partie de la vulnérabilité. Philip Pettit se fait l'avocat de la première option, et conçoit la non-domination (c'est-à-dire la possibilité de vivre en l'absence d'interférences arbitraires) comme une forme de pouvoir. «Elle représente une capacité de contrôle que possède une personne sur sa propre destinée, et une forme de pouvoir qui nous est familière: le pouvoir d'un agent qui peut empêcher que certains torts lui soient causés $»^{28}$. Cela se traduit par l'importance qu'il conferre à l'idée de contestabilité comme critère permettant d'attester du caractère non-arbitraire d'une interférence, c'est-à-dire à la possibilité, pour le sujet concerné, d'actualiser un pouvoir de révision du monde. Il s'agit par là de récupérer une forme de maîtrise face à la fragilité de mes actions et de mes énoncés dans un monde qui n'est pas de mon fait. La seconde possibilité est par exemple défendue par Robert Goodin, en l'espèce d'une responsabilité collective qu'il y a, dans le cas d'une vulnérabilité de $\mathrm{A}$ aux actions d'un groupe, à organiser et implémenter un schéma d'action coordonnée tel que les intérêts de A soient protégés par ce groupe, ce qui implique, pour chacun de ses membres, une responsabilité à la fois de s'assurer que le schéma d'action est effectivement réalisé, et de remplir les responsabilités spécifiques qui lui sont allouées dans le cadre de cette organisation ${ }^{29}$.

Dans cette littérature abondante sur une vulnérabilité constitutive, on peut ainsi établir trois ensembles thématiques, qui renvoient à des logiques éthiques, et des solutions politiques, différentes. Partageant une conception du sujet radicalement alternative, elles composent en outre un horizon politique commun, qui est tissé par leur propre assertion.

Elles évoquent en effet souvent l'effet incapacitant, sur la personne vulnérable, de la conscience qu'elle peut avoir de la vulnérabilité de ses actes et de son corps. Pettit en a fait un élément capital de la domination, tandis que Gilligan posait: "C'est un sens de la vulnérabilité qui empêche les femmes de prendre position, ce que George Eliot nomme la «sensibilité» de la fille aux jugements négatifs des autres, qui dérive

26 Martha Nussbaum, Femmes et développement humain, Paris, Éditions des femmes, 2008, p. 29.

27 Robert Goodin, Protecting the Vulnerable, op. cit., p. 197-200.

28 Philip Pettit, Républicanisme, op. cit., p. 97.

29 Robert Goodin, Protecting the Vulnerable, op. cit., p. 136. 


\section{Majeure Politiques du care}

de son manque de pouvoir et son incapacité subséquente à faire quelque chose dans le monde ${ }^{30}$ Préserver notre capacité à être l'auteur de nos vies semble alors impliquer l'oubli ou le déni de notre vulnérabilité; l'apparition contemporaine de ces théories, qui posent comme générale l'expérience de vivre à la merci d'autrui, peut avoir une force politique rendre inutile un tel oubli. On peut faire l'hypothèse que le sens de sa propre vulnérabilité n'est incapacitant que parce qu'il ne surgit que chez certains d'entre nous. Généraliser la certitude de cette vulnérabilité constitutive représente alors le premier pas en direction de l'avènement d'une seule classe de vulnérabilité. 